\title{
Thymoquinone Promotes Pancreatic Cancer Cell Death and Reduction of Tumor Size through Combined Inhibition of Histone Deacetylation and Induction of Histone Acetylation
}

\author{
Daniel Relles, ${ }^{1}$ Galina I. Chipitsyna, ${ }^{2}$ Qiaoke Gong, ${ }^{1}$ Charles J. Yeo, ${ }^{1}$ and Hwyda A. Arafat ${ }^{2}$ \\ ${ }^{1}$ Departments of Surgery, Jefferson Pancreatic, Biliary and Related Cancer Center, Thomas Jefferson University, Philadelphia, PA, USA \\ ${ }^{2}$ Department of Biomedical Sciences, University of New England, Biddeford, ME, USA
}

Correspondence should be addressed to Galina I. Chipitsyna; galina727@gmail.com

Received 23 March 2016; Revised 29 August 2016; Accepted 23 November 2016

Academic Editor: William C. Cho

Copyright (C) 2016 Daniel Relles et al. This is an open access article distributed under the Creative Commons Attribution License, which permits unrestricted use, distribution, and reproduction in any medium, provided the original work is properly cited.

\begin{abstract}
Pancreatic ductal adenocarcinoma (PDAC) is virtually therapy-resistant. As noninvasive lesions progress to malignancy, the precursor period provides a window for cancer therapies that can interfere with neoplastic progression. Thymoquinone (Tq), a major bioactive component of essential oil from Nigella sativa's seeds, has demonstrated antineoplastic activities in multiple cancers. In this study, we investigated antineoplastic potential of Tq in human PDAC cell lines, AsPC-1 and MiaPaCa-2. Tq (10-50 $\mu \mathrm{M}$ ) inhibited cell viability and proliferation and caused partial G2 cycle arrest in dose-dependent manner in both cell lines. Cells accumulated in subG0/G1 phase, indicating apoptosis. This was associated with upregulation of p53 and downregulation of Bcl-2. Independently of p53, Tq increased p21 mRNA expression 12-fold. Tq also induced $\mathrm{H} 4$ acetylation (lysine 12) and downregulated HDACs activity, reducing expression of HDACs 1, 2, and 3 by $40-60 \%$. In vivo, Tq significantly reduced tumor size in $67 \%$ of established tumor xenografts $(P<0.05)$, along with increased $\mathrm{H} 4$ acetylation and reduced HDACs expression. Our results showed that $\mathrm{Tq}$ mediated posttranslational modification of histone acetylation, inhibited HDACs expression, and induced proapoptotic signaling pathways. These molecular targets demonstrate rationale for using $\mathrm{Tq}$ as a promising antineoplastic agent to prevent postoperative cancer recurrence and to prolong survival of PDAC patients after surgical resection.
\end{abstract}

\section{Introduction}

Nigella sativa is a herb used in traditional medicine in many Middle Eastern countries to treat a broad array of diseases, including asthma, diabetes, hypertension, headache, eczema, and various gastrointestinal ailments. Thymoquinone (Tq), the most abundant constituent of Nigella sativa seed's essential oil extract, has been demonstrated to have antioxidant and anti-inflammatory activity through mechanisms that are still being defined [1-3]. Additionally, Tq has been reported to display antineoplastic activity in multiple cancers, including prostate, colorectal, breast, sarcoma, and leukemia [1-3]. Tq has been shown to inhibit cancer cells proliferation, arrest cancer cell cycle progression, induce proapoptotic effects, and enhance cytotoxic activity of chemotherapeutic agents [3-6]. Implicated molecular targets include $\mathrm{Bcl}-2$, caspases, PPARs, NF-kB, STAT3, MAPK, Akt, and ROS [3]. Proapoptotic activity of $\mathrm{Tq}$ is revealed through p53-dependent and independent pathways. In murine model xenografts $\mathrm{Tq}$ has been shown to suppress tumor growth, likely through angiogenesis inhibition [4]. Tq was shown to inhibit cell migration and invasion in breast cancer cells in a dose-dependent manner [3]. Finally, Tq has been shown to mitigate some of the drugrelated toxicities associated with several chemotherapeutic agents $[3,5,6]$. Small study of safety and clinical activity of $\mathrm{Tq}$ in humans found no adverse effects with doses up to $2600 \mathrm{mg} /$ day [3].

In eukaryotic cells, DNA is tightly wound to form nucleosomes around core histone proteins. Regulation of gene expression is controlled through acetylation and deacetylation of histones, which are modified in posttranslational manner through histone acetyltransferases (HATs) and histone deacetylases (HDACs). Inhibition of HDACs leads to histones acetylation and subsequent changes in gene expression, 
and it has been shown to inhibit cell cycle progression and promote apoptosis in cancer cells [7]. HDAC inhibitors have been reported to induce p21 expression in p53-independent manner [8]. We recently have shown that $\mathrm{Tq}$ can act as a novel inhibitor of proinflammatory pathways in PDAC cells, and this is mediated through significant downregulation of constitutive and TNF- $\alpha$-activated NF- $\kappa \mathrm{B}$ [9]. Similar results were obtained using Trichostatin A (TSA), a well-established HDAC inhibitor, thus showing that $\mathrm{Tq}$ can act as HDAC inhibitor [9]. However, it is yet to be determined whether Tq's antineoplastic activity involves histone acetylation and deacetylation.

In this study, we explored the antineoplastic potential of $\mathrm{Tq}$ in PDAC cells. In addition to studies investigating the relationship between $\mathrm{Tq}$ and $\mathrm{p} 21$ expression, we evaluated Tq's effect on histone acetylation/deacetylation in vitro and in vivo.

\section{Materials and Methods}

2.1. Cell Culture. PDAC cell lines, AsPc-1, MiaPaCa-2, and Hs766T, were purchased from ATCC. Cells were cultured in DMEM supplemented with $10 \%$ fetal bovine serum in humid atmosphere of $5 \% \mathrm{CO}_{2}$. Cells were treated with Tq (10$50 \mu \mathrm{M})$ (Sigma-Aldrich). Tq concentrations were selected based on dose-response studies.

2.2. MTT Assay. Cells were grown in 48-well plates and incubated in full growth media at $37^{\circ} \mathrm{C}$ and $5 \% \mathrm{CO}_{2}$. After treatment with $\mathrm{Tq}$, cell viability was examined using MTT (Methylthiazolyldiphenyl-tetrazolium bromide) conversion assay as previously described. Briefly, MTT (Sigma-Aldrich) was added to the wells $(500 \mu \mathrm{g} / \mathrm{mL})$. After 4 hours formazan crystals were solubilized in DMSO, and the optical density was measured at $570 \mathrm{~nm}$. Optical density directly correlated with viable cells quantity. Experiments were made in triplicate and repeated 3 times.

2.3. Flow Cytometric Analysis of DNA Content. Cells, seeded in $100 \mathrm{~mm}$ dishes at a density of $7.5 \times 10^{5}$ cells/dish, were treated with $\mathrm{Tq}$ and incubated for 24 or $48 \mathrm{~h}$. Cells were harvested, washed with phosphate-buffered saline (DPBS), permeabilized with $70 \%$ ethanol, treated with $1 \%$ RNase, and stained with propidium iodide (PI) at $100 \mu \mathrm{g} / \mathrm{mL}$. Flow cytometric analyses were carried out in a fluorescenceactivated sorter (FACStar plus; Becton Dickinson) with a $360 \mathrm{~nm}$ Argon-Iron laser. 10,000 events per sample were collected and analyzed using the Cell Fit analysis program (Becton Dickinson).

2.4. Annexin V-FITC Staining. After treatment with $\mathrm{Tq}$, cells were washed with DPBS buffer and removed from the growth plates. Cells were collected by centrifugation at $500 \mathrm{~g}$ for $5 \mathrm{~min}$, and Annexin V-FITC reagent (BD Bioscience) with or without PI counterstain was used to resuspend the pellet. After incubation in darkness for $15 \mathrm{~min}$ at room temperature cells were analyzed by flow cytometry. FITC (FL1, BP525/5 nm) and PI (FL3, BP 610/5 nm) were collected in logarithmic mode. Analysis was performed using Beckman Coulter Elite software.
2.5. RNA Extraction and $q R T-P C R$. Total RNA was isolated using Tri-Reagent (Life Technologies). RNAs were quantified and cDNAs were synthesized using ImProm-II ${ }^{\mathrm{TM}}$ Reverse Transcription System (Promega). TaqMan gene expression assays were purchased from Applied Biosystems. GAPDH was used as a housekeeping gene. cDNAs were subjected to real time qPCR using HotStart-IT or VeriQuest Probe qPCR Master Mixes (Affymetrix) and TaqMan technology (7500 Sequence Detector, Applied Biosystems). The relative mRNA levels were quantified using Applied Biosystems software.

2.6. Protein Isolation and Western Blot Analysis. Cell or tissue lysates were analyzed as described elsewhere [10]. Cells were lysed in modified RIPA lysis buffer, and protein concentrations in the supernatant were determined using BCA protein assay reagent (Pierce). Equal protein quantities $(25 \mu \mathrm{g})$ were run in $10 \%$ SDS-polyacrylamide slab gels, transferred to polyvinylidene difluoride membranes, and incubated with primary antibodies, acetylated H4 (Active motif), and anti- $\beta$ actin (Chemicon), both diluted in PBST. After incubation with secondary antibodies, protein bands were visualized with enhanced chemiluminescence reagents (ECL Plus Western Blotting Detection System, Amersham Pharmacia Biotech).

2.7. Histone Deacetylases (HDACs) Activity. To determine HDACs activity in PDAC cells after Tq treatment we used a colorimetric HDAC activity assay kit (Biovision). HDAC colorimetric substrate (acetylated lysine side chain) was incubated with nuclear extract of PDAC cells after treatment with Tq. Deacetylation of the substrate sensitized the substrate, which upon treatment with the Lysine Developer produced a chromophore. The chromophore absorbance at $405 \mathrm{~nm}$ that directly correlated with HDAC activity was determined using Synergy HT multidetection microplate reader (BioTeck).

2.8. Tq Toxicity in Mice. Mice (8-week-old) were divided into groups of 10 each and $\mathrm{Tq}$ toxicity was determined after daily intraperitoneal injections for 20 consecutive days. The doses of $\mathrm{Tq}$ were $5,10,20$, or $30 \mathrm{mg} / \mathrm{kg}$ body weight. Tq dilutions in isotonic saline $(0.9 \%)$ were prepared and control animals received the same percentage of ethanol diluted in saline.

2.9. Xenograft Model. Animal studies were performed after approval of the protocol by IACUC at Thomas Jefferson University Hospital. Xenograft model for PDAC was generated in 4-week-old male nude mice ( $\mathrm{Crl}: \mathrm{Nu} / \mathrm{Nu}$-muBR) weighing 20-22 g (Charles River Laboratories). Suspensions of AsPC- 1 or Hs766T PDAC cells $\left(5 \times 10^{6}\right.$ in $0.2 \mathrm{~mL}$ PBS $)$ were injected subcutaneously into the flank of 4-6-week-old male mice. Animals were kept in light- and temperature-controlled environment and provided with food and water ad libitum. Tumor size was determined daily. Intraperitoneal treatment with $\mathrm{Tq}(5-30 \mathrm{mg} / \mathrm{kg}$ ) or $0.1 \%$ methanol in physiologic saline three times per week was started when subcutaneous tumors reached a diameter of $1 \mathrm{~cm}$. After 5 weeks animals were sacrificed, tumor sizes were measured, and tumors were processed for evaluation of HDAC expression levels by real time qPCR. 


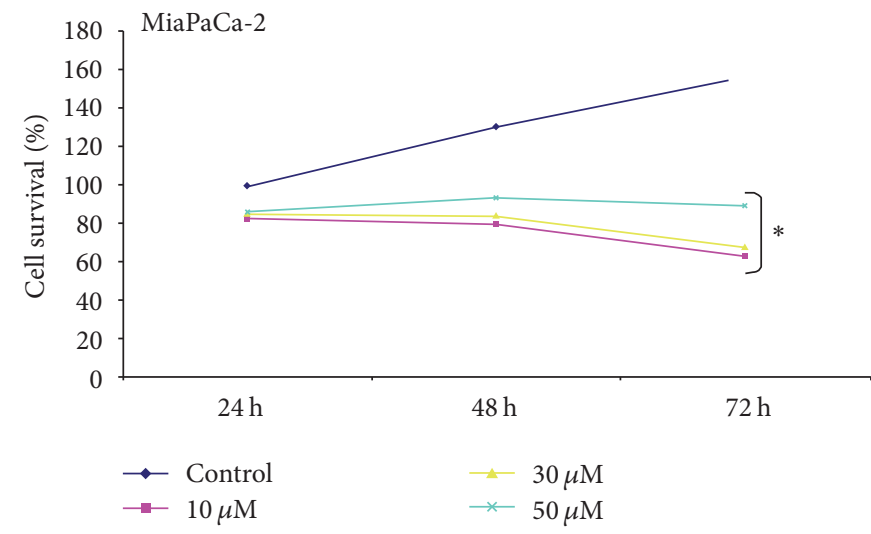

(a)

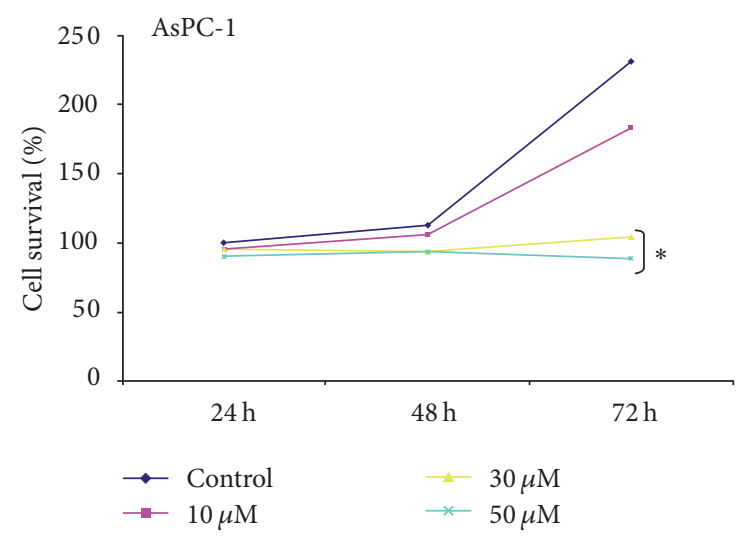

(b)

Figure 1: Effect of Tq on PDAC cell proliferation. (a) MiaPaCa-2 and (b) AsPc-1 cells were exposed to Tq (10-50 $\mu \mathrm{M})$ during 24, 48, and 72 h. ${ }^{*} P<0.05$ versus control, using one-way repeated ANOVA with subsequent all pairwise comparison procedure by Student's $t$-test.

2.10. Statistical Analysis. All experiments were performed 4 to 6 times. Data were analyzed for statistical significance by ANOVA with post hoc Student's $t$-test analysis. These analyses were performed with assistance of a computer program (JMP 5 Software SAS Campus Drive). Differences were considered significant at $P \leq 0.05$. Tumor sizes were estimated for significant differences by the Manny-Whitney rank sum test.

\section{Results}

3.1. Tq Induces a Dose-Dependent Reduction of PDAC Cell Growth. Cell viability was significantly reduced by $\mathrm{Tq}$ as a function of both concentration and time, in both cell lines, MiaPaCa-2 (Figure 1(a)), and AsPC-1 (Figure 1(b)). For example, cell survival for MiaPaCa-2 cells treated with $50 \mu \mathrm{M}$ Tq for 24,48 , and $72 \mathrm{~h}$ was $82 \%, 80 \%$, and $62 \%$, respectively. Differences in cell survival at increasing concentrations became more pronounced with longer treatment times. These results confirm that $\mathrm{Tq}$ inhibits $\mathrm{PDAC}$ cell proliferation.

3.2. Tq Induces Accumulation of PDAC Cells in the PreG1 Peak. Cell cycle profiles were monitored by flow cytometric analysis of PI stained cellular DNA content at 24 and $48 \mathrm{~h}$ after treating MiaPaCa-2 and AsPC-1 cells with Tq (30 and $50 \mu \mathrm{M})$. DNA histograms show evident accumulation of hypodiploid cells at preG1 peak within $24 \mathrm{~h}$ indicating accumulation of dead and apoptotic cells. At $30 \mu \mathrm{M}$ of Tq the percentage of apoptotic cells increased to $29 \%$ at $24 \mathrm{~h}$ and reached $49 \%$ at $48 \mathrm{~h}$ (Figure 2(a)), while it increased to $15 \%$ in AsPC-1 cells after $24 \mathrm{~h}$ and reached $24 \%$ in after $48 \mathrm{~h}$ (Figure $2(\mathrm{~b})$ ).

3.3. Tq Induces Late Apoptosis. Analysis of the number of cells in the histogram revealed that the number of late apoptotic cells was significantly increased with concomitant reduction in the number of live cells in both MiaPaCa-2 (Figure 3(a)) and AsPC-1 cells (Figure 3(b)). These findings confirmed that Tq-induced PDAC cell number increases in preG1 fraction were due to apoptosis.
3.4. Tq-Induced Apoptosis Is Accompanied by Regulation of Apoptosis-Related Genes. To analyze the molecular mechanisms of Tq-mediated apoptosis, we explored the expression of pro- and antiapoptotic genes in $\mathrm{MiaPaCa}-2$ cells. Real time qPCR analysis indicated that $\mathrm{Tq}$ induced a dose-dependent increase in Bax mRNA expression (Figure 4). Tq dosedependently downregulated Bcl-2 mRNA expression and, as a result, increased $\mathrm{Bax} / \mathrm{Bcl}-2$ mRNA ratio. In addition, $\mathrm{Tq}$ induced 2-fold increase in p53 mRNA expression after $24 \mathrm{~h}$ of treatment and 12-fold increase in p21 mRNA expression after as early as $3 \mathrm{~h}$ of treatment (Figure 4). Cells were also pretreated with p53 inhibitor, PFT- $\alpha$, for $1 \mathrm{~h}$ prior to addition of Tq. This treatment abolished p53 induction, decreased $\mathrm{Bax} / \mathrm{Bcl}-2$ mRNA ratio, and reduced expression of p $21 \mathrm{mRNA}$ from 12 -fold to 2-3-fold. These data provided the evidence of p53-independent upregulation of p21 by Tq. Since many HDAC inhibitors have been shown to stimulate p21 signaling regardless of the mutation status of $\mathrm{p} 53$, we investigated whether Tq can act as HDAC inhibitor.

3.5. Tq Significantly Reduces the Expression of HDACs 1, 2, and 3 mRNA Expression and HDAC Activity. To determine whether $\mathrm{Tq}$ acts through inhibition of HDACs, we evaluated the expression of HDACs $1-6$ by real time qPCR. As seen in Figure $5, \mathrm{Tq}(50 \mu \mathrm{M})$ reduced the expression of HDACs 1-3 by $40-50 \%$. Tq $(50 \mu \mathrm{M})$ time-dependently reduced HDACs activity by $60 \%$ (Figure 6).

3.6. Tq Dose-Dependently Increases Histone Acetylation. To assess whether histone acetylation is involved in $\mathrm{Tq}$ mode of action, we evaluated the acetylation of lysine 12 in histone 4 (H4 Ac-K12) by Western blotting. Nuclear extracts from Tq treated (30-50 $\mu \mathrm{M}) \mathrm{MiaPaCa}-2$ cells showed dose-dependent increased acetylation after $24 \mathrm{~h}$ (Figure 7). These data indicate that $\mathrm{Tq}$ not only reduces HDAC expression and activity but also increases histone acetylation.

3.7. Tq Significantly Reduces Tumor Size in Human PDAC Xenografts. Next, we tested the effect of different doses of Tq 
$\mathrm{Tq}(\mu \mathrm{M})$
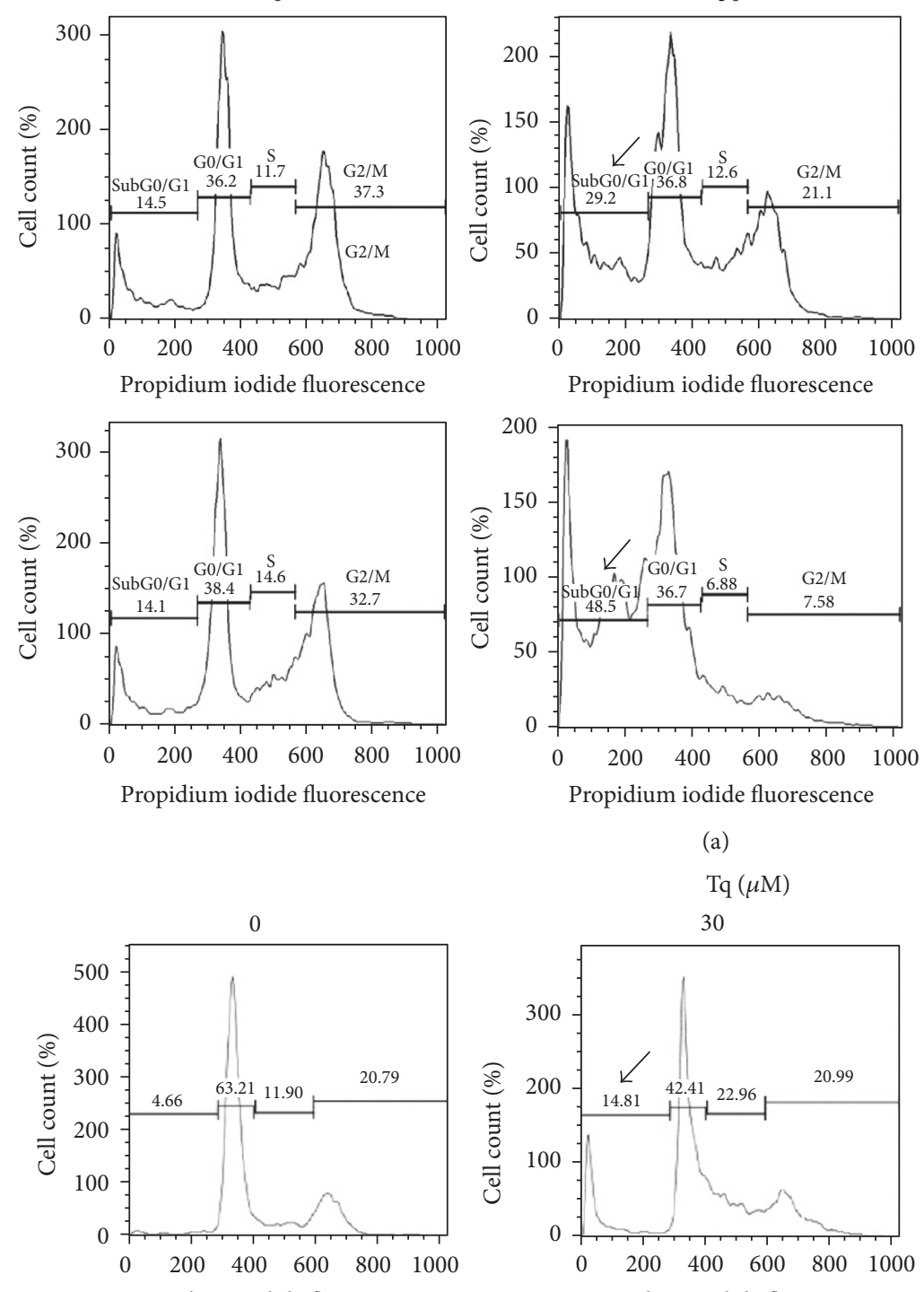

Propidium iodide fluorescence

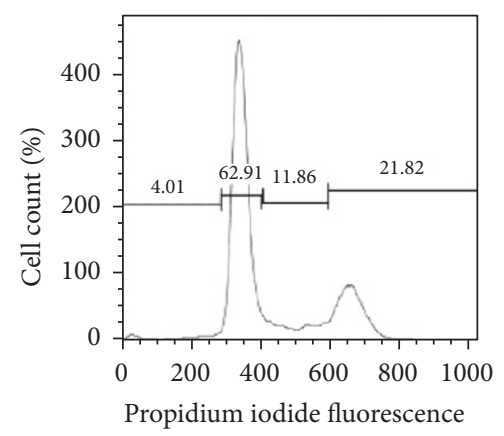

(a)

$\mathrm{Tq}(\mu \mathrm{M})$

30

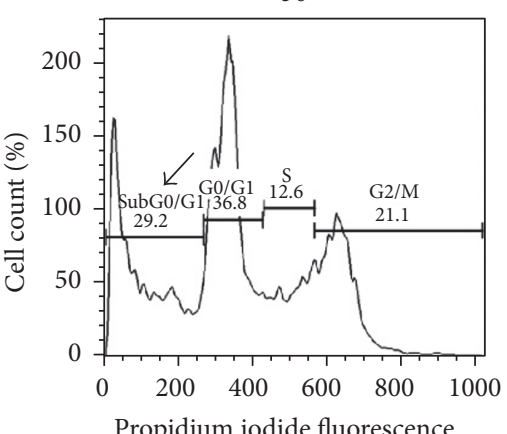

30
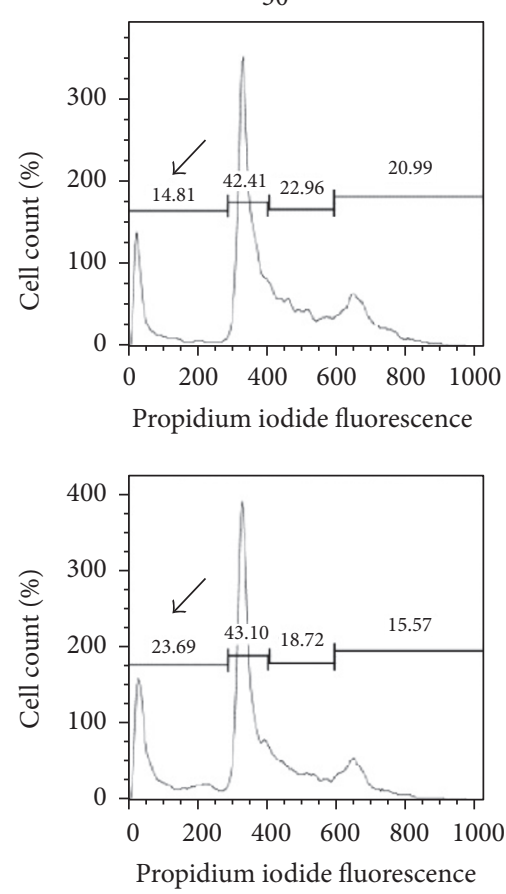

50
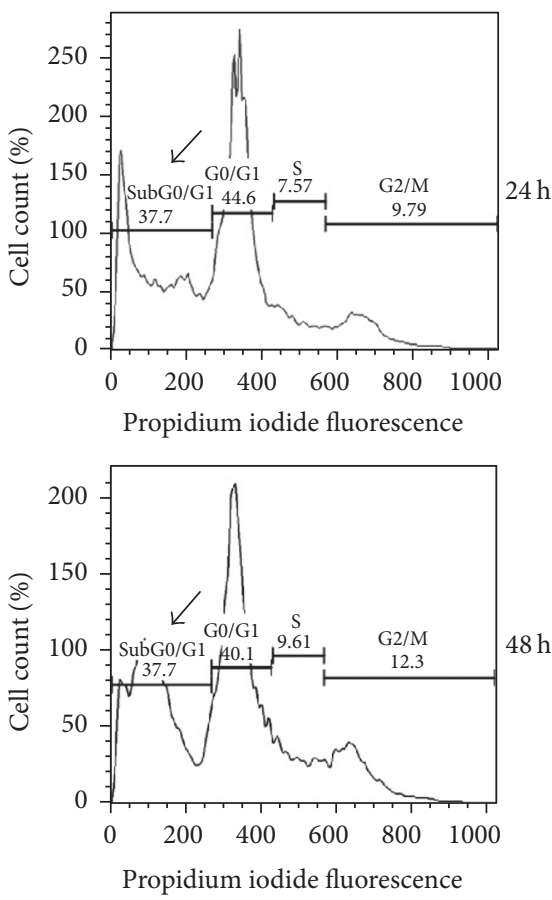

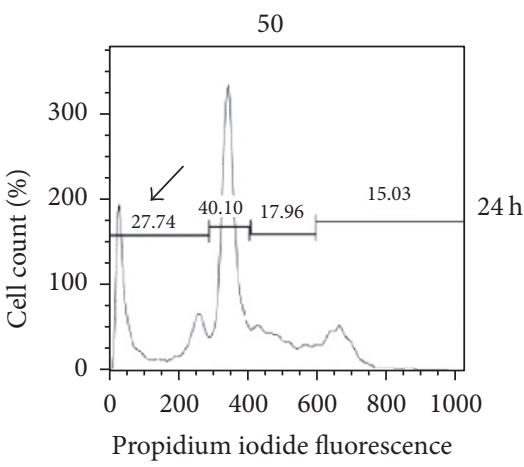

(b)

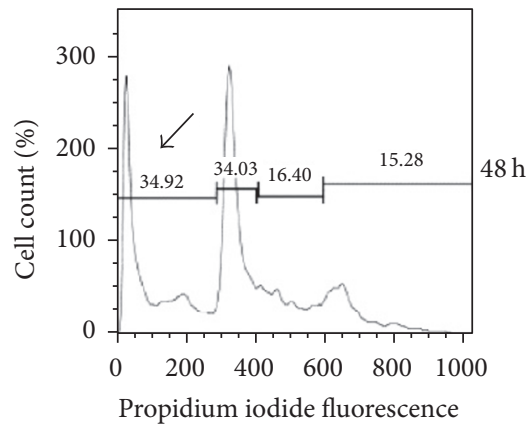

FIGURE 2: Cell cycle profiles by flow cytometry: (a) MiaPaCa-2 and (b) AsPc-1 cells. Tq induced accumulation of the cells in preG1 phase. The data shown are typical of one of three independent experiments. DNA histograms show evident accumulation of hypodiploid cells at preG1 peak within $24 \mathrm{~h}$ indicating accumulation of dead and apoptotic cells. At $30 \mu \mathrm{M}$ of Tq the percentage of apoptotic cells increased to $29 \%$ at $24 \mathrm{~h}$ and reached $49 \%$ at $48 \mathrm{~h}$ (a), while it increased to $15 \%$ in AsPC-1 cells after $24 \mathrm{~h}$ and reached $24 \%$ after $48 \mathrm{~h}$ (b). Data were quantified from three experiments, ${ }^{*} \mathrm{P}<0.05$, using one-way repeated ANOVA with subsequent all pairwise comparison procedure by Student's $t$-test. 
$\mathrm{Tq}(\mu \mathrm{M})$
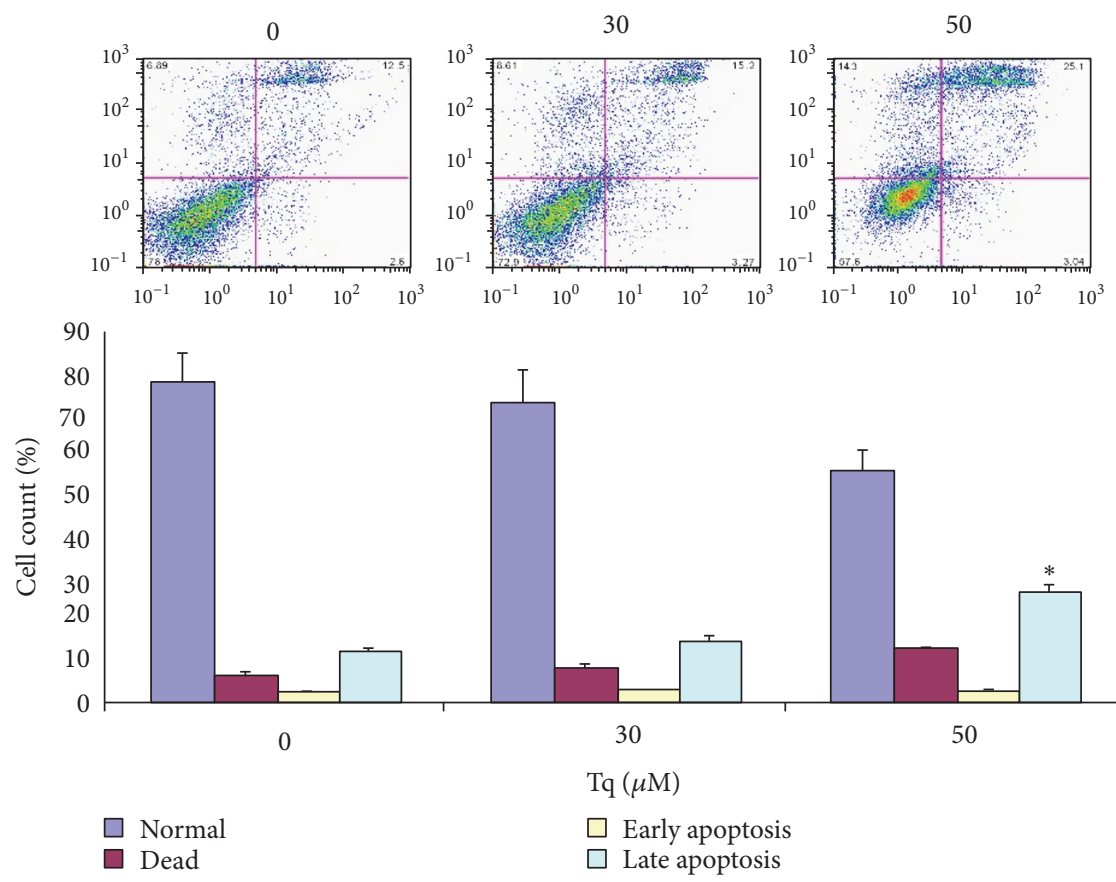

(a)

$\mathrm{Tq}(\mu \mathrm{M})$
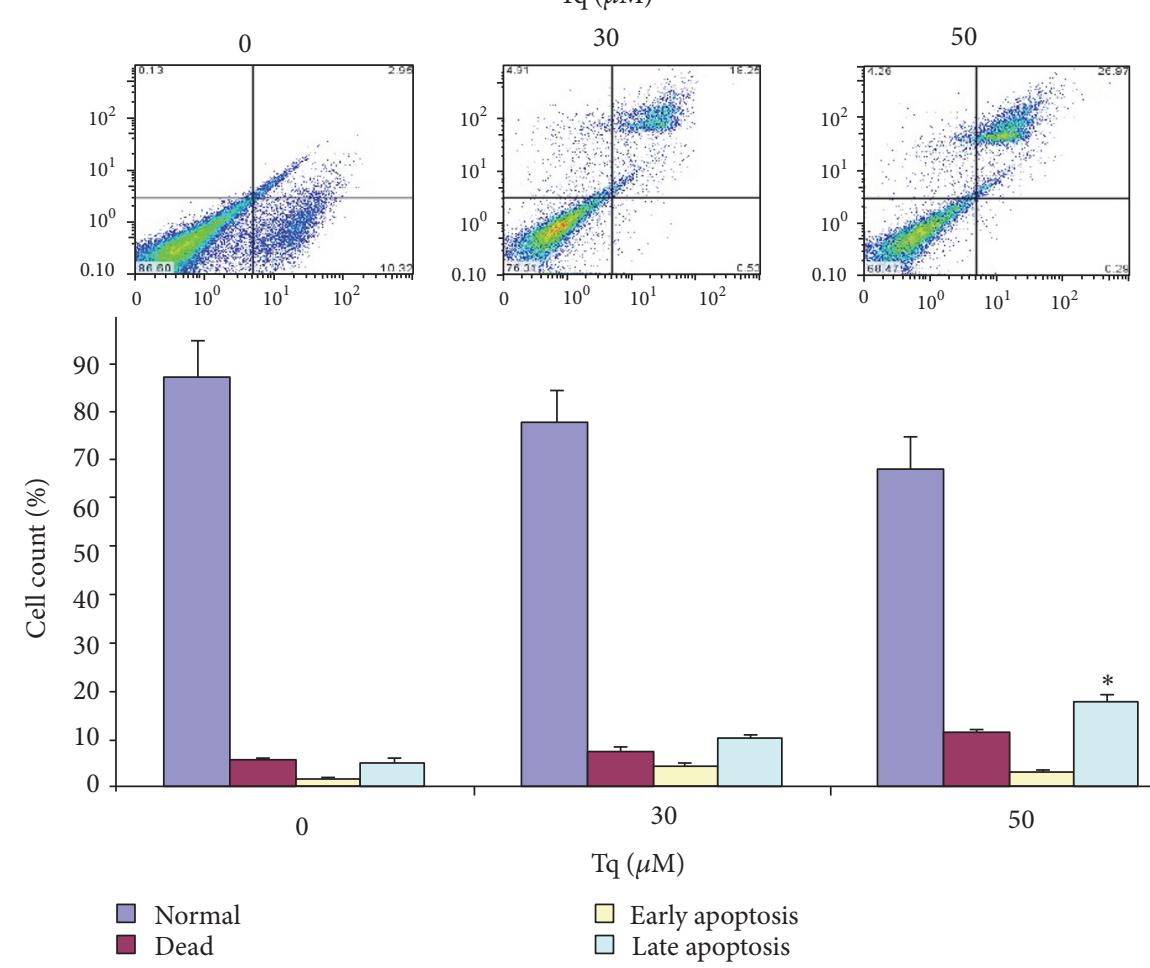

(b)

Figure 3: Apoptosis in Tq-induced PreG1 fraction. (a) MiaPaCa-2 and (b) AsPc-1 cells were treated with Tq (30 and $50 \mu \mathrm{M}$ ) for $24 \mathrm{~h}$, stained with Annexin V and PI, and apoptosis was analyzed by flow cytometry. The upper right quadrants represent apoptotic cells, Annexin V positive and PI negative. Quantification of cell numbers in histogram shows significant increase in the number of late apoptotic cells. Data were quantified from four experiments ${ }^{*} P<0.05$, using one-way repeated ANOVA with subsequent all pairwise comparison procedure by Student's $t$-test. 


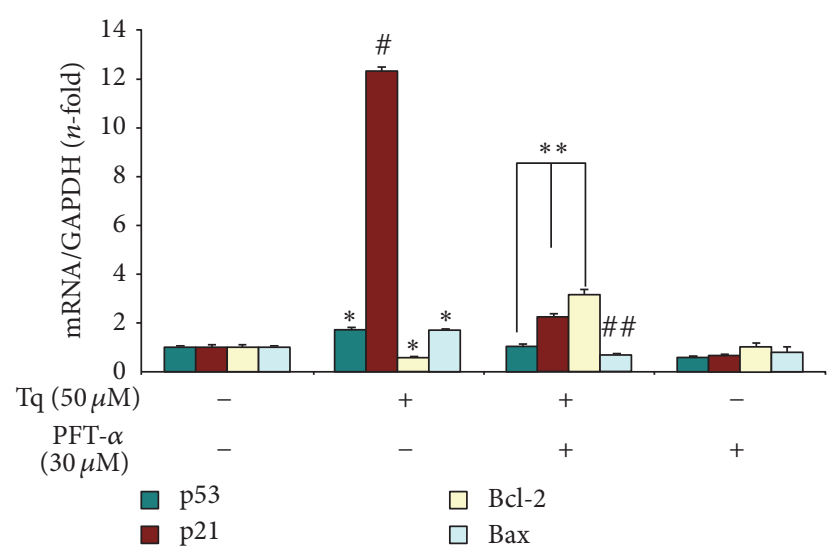

FIgURE 4: Real time qPCR analysis shows that $\mathrm{Tq}(50 \mu \mathrm{M})$ significantly increased p53, p21, and Bax mRNA expression and downregulated Bcl-2 mRNA expression in MiaPaCa-2 cells. Cells were also pretreated with $\mathrm{p} 53$ inhibitor, $\operatorname{PFT}-\alpha$, for $1 \mathrm{~h}$ prior to addition of Tq. Values are expressed as mean \pm SEM of three experiments. ${ }^{*} P<0.05{ }^{\#} P<0.02$ versus control levels ${ }^{* *} P<$ $0.05{ }^{\# \#} P<0.02$ versus Tq treated values, using one-way repeated ANOVA with subsequent all pairwise comparison procedure by Student's $t$-test.

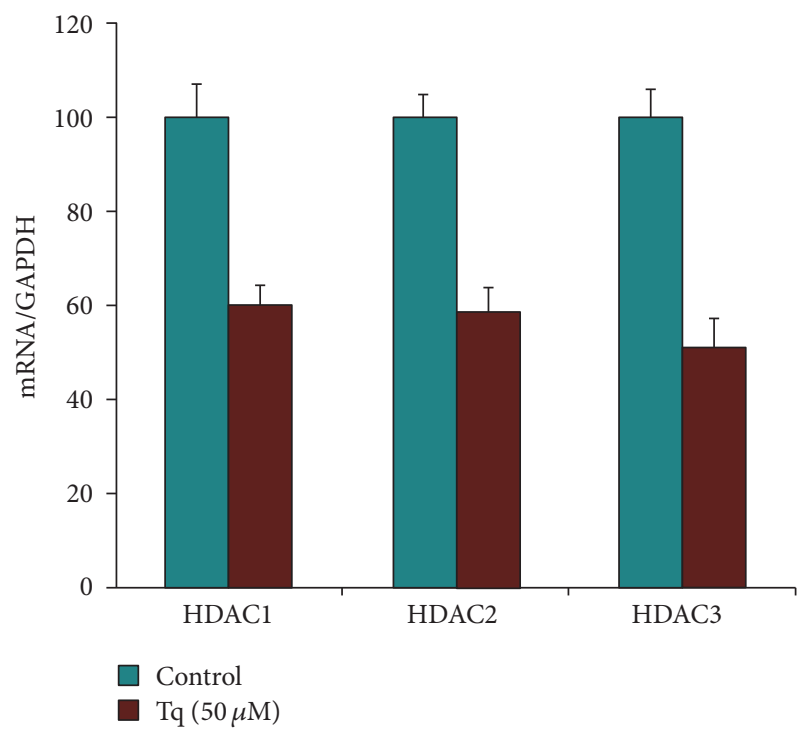

Figure 5: Real time qPCR analysis of MiaPaCa-2 cells showing significant reduction of HDACs 1, 2, and 3 levels upon treatment with $\mathrm{Tq}(50 \mu \mathrm{M}) .{ }^{*} P<0.05$ versus control levels, using oneway repeated ANOVA with subsequent all pairwise comparison procedure by Student's $t$-test.

in human PDAC xenografts. Xenografts were generated in 4-week-old male nude mice $(\mathrm{Crl}: \mathrm{Nu} / \mathrm{Nu}$-muBR) weighing 20-22 g (Charles River laboratories). Human PDAC cell lines (AsPC-1 and Hs766T) were utilized. When the tumors reached $1 \mathrm{~cm}$, animals were treated with different doses of Tq (5-30 $\mathrm{mg} / \mathrm{kg}$ body weight). We show here data from animals that were treated with $\mathrm{Tq}$ at $30 \mathrm{mg} / \mathrm{kg}$ body weight for 5 weeks. Tumor size was significantly $(P<0.05)$ shrunken in $67 \%$ of the animals (Figure 8). Analysis of HDACs in the tumors

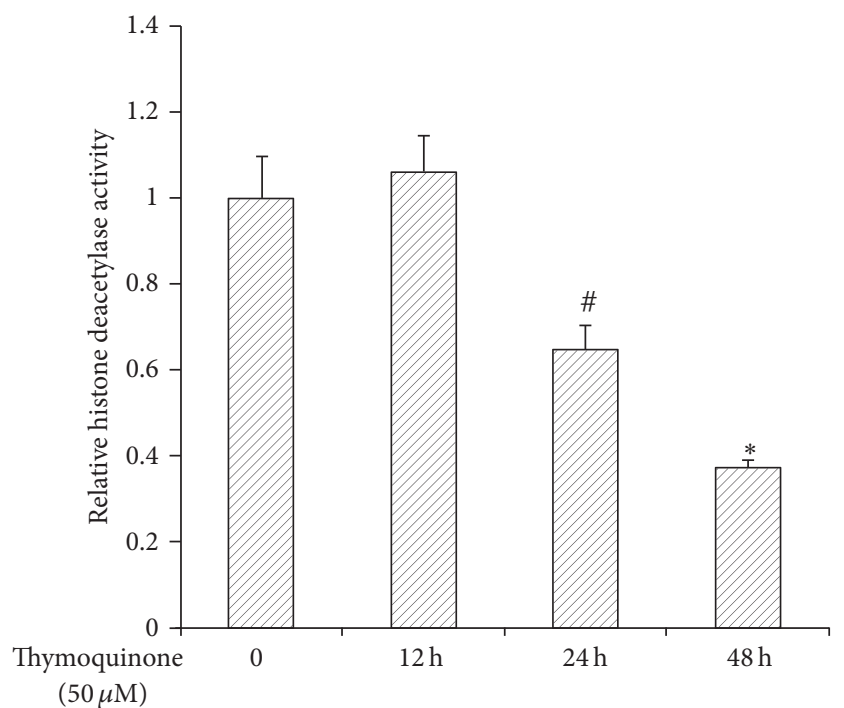

Figure 6: MiaPaCa-2 cells were treated with or without $\mathrm{Tq}(50 \mu \mathrm{M})$ for 12-48 h. HDACs activity decreased significantly at 24 and $48 \mathrm{~h}$. Values are expressed as mean \pm standard deviation of three different observations. ${ }^{\#} P<0.05{ }^{*} P<0.002$ versus control levels, using one-way repeated ANOVA with subsequent all pairwise comparison procedure by Student's $t$-test.

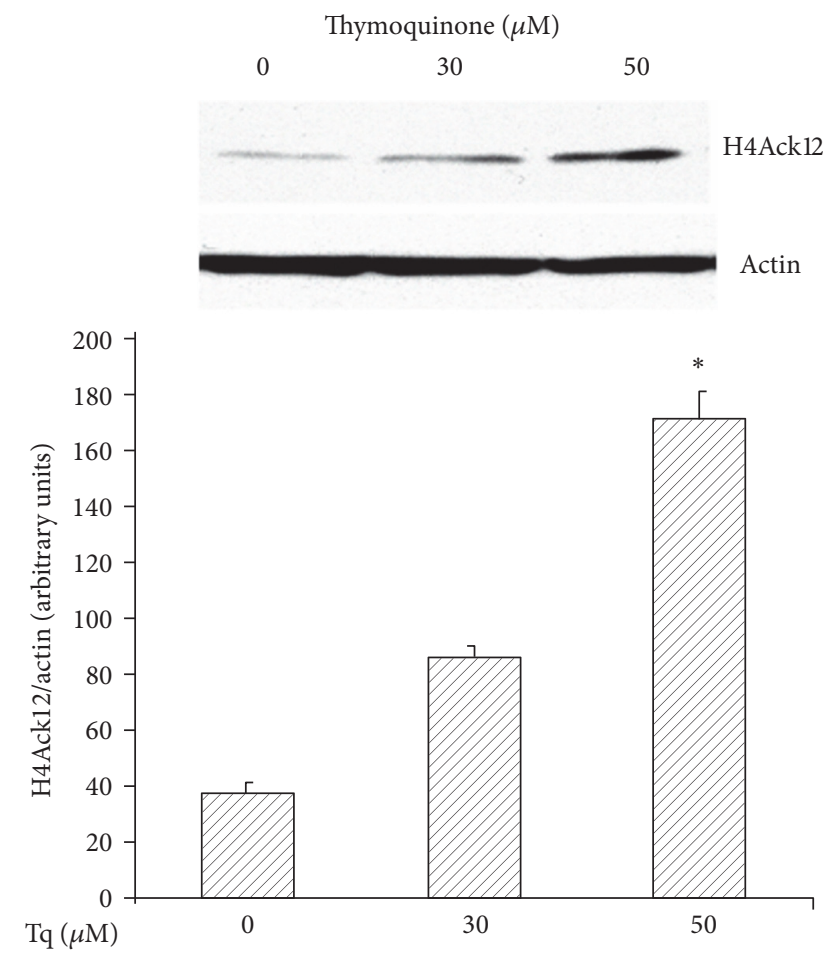

FIGURE 7: Representative Western immunoblot of nuclear extracts from MiaPaCa-2 cells. H4 Ac K12 protein is expressed as $8 \mathrm{kDa}$ band. Significant increase of $\mathrm{H} 4 \mathrm{Ac} \mathrm{K} 12$ protein is seen in cells treated with Tq $(30-50 \mu \mathrm{M})$ after $48 \mathrm{~h}$. Data are means \pm SEM. ${ }^{*} P<0.05$ versus control untreated cells. 


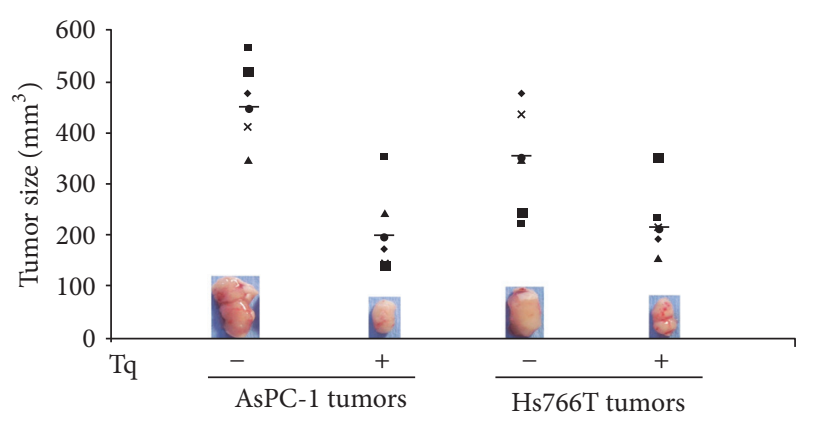

FIGURE 8: Xenografts generated in 4-week-old male nude mice using AsPC-1 and Hs766T cells. When tumors reached $1 \mathrm{~cm}$, animals were treated with $\mathrm{Tq}(30 \mathrm{mg} / \mathrm{kg}$ body weight i.p. for 5 weeks). Tumor size was significantly $(P<0.05)$ shrunken in $67 \%$ of the animals.

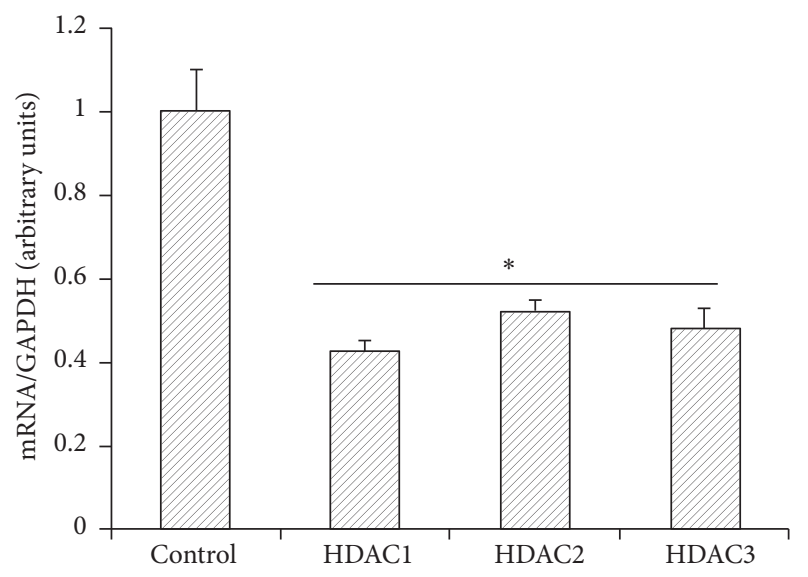

FIGURE 9: Real time qPCR of Tq treated xenografts showing significant reduction of HDACs 1, 2, and 3 mRNA expression in AsPC-1 cells. Data are means \pm SEM. ${ }^{*} P<0.05$ versus control untreated tumors.

indicated that $\mathrm{Tq}$ had significantly reduced the expression of HDACs 1, 2, and 3 mRNA when compared to vehicle-treated controls (Figure 9).

\section{Discussion}

Pancreatic ductal adenocarcinoma (PDAC) is still an urgent clinical problem because of its aggressive nature and resistance to chemotherapy and radiation. In this study, we show that thymoquinone (Tq), the active ingredient of the Middle Eastern herb, Nigella sativa, significantly inhibits PDAC cell growth and triggers induction of different proapoptotic signaling pathways in human PDAC cells in vitro and in vivo. This study is also the first to show that $\mathrm{Tq}$ mediates posttranslational modification of histone acetylation and inhibition of HDACs expression.

Tumor suppressor gene p53 is a sensor of cellular stress and is a critical activator of intrinsic pathway of apoptosis. Stimulation of p53 can initiate apoptosis by activating proapoptotic $\mathrm{Bcl}-2$ family member, such as Bax, and repressing antiapoptotic Bcl-2 proteins, such as Bcl2 and $\mathrm{Bcl}-\mathrm{xl}$. Bax undergoes conformational changes and subsequently translocates to mitochondria, where it inserts into the outer membrane as oligomers, resulting in the release of cytochrome $c$ and apoptosis [11]. Bcl-2/Bcl-xl form heterodimers with Bax and prevent its insertion into the mitochondrial membrane. Therefore, the ratio of $\mathrm{Bcl}-2 / \mathrm{Bcl}-$ $\mathrm{xl}$ to Bax is critical for the determination of the apoptotic threshold. Studies have shown that deregulation of Bax/Bcl$2 / \mathrm{Bcl}$-xl contributes to the development, growth, and expansion of PDAC [12], and increased Bax and reduced Bcl-2/Bcl$\mathrm{xl}$ significantly increase the sensitivity to chemotherapeutic drugs $[13,14]$. Bcl-xl is believed to be an ideal target for PDAC therapy as it is constitutively overexpressed in PDAC cell lines that are highly resistant to Fas- and TRAIL-mediated apoptosis [15]. In our studies, we showed that Tq $(100 \mu \mathrm{M})$ induced a significant increase in $\mathrm{Bax} / \mathrm{Bcl}-2 \mathrm{mRNA}$ expression ratio and caused apoptosis in PDAC cells.

Cells also respond to activation of p53 tumor suppressor by undergoing cell cycle arrest through activation of p21 expression. Our results demonstrated that there was a significant increase (12-fold) in p21 expression, without an equivalent increase in p53 expression (2-fold) in PDAC cells treated with $\mathrm{Tq}$ for as early as $3 \mathrm{~h}$. This suggests that $\mathrm{Tq}$ induces p21 expression, partially in p53-independent manner. Since many HDAC inhibitors have been shown to stimulate p21 signaling regardless of the mutation status of p53 $[8,16]$, we investigated whether $\mathrm{Tq}$ could act as HDAC inhibitor.

Histone acetylation and deacetylation (epigenetic regulation) is the mechanism of regulating gene expression. In tumor cells, acetylation/deacetylation balance becomes disrupted. As histones are modified chromatin structure and subsequent gene expression change. Histone acetyltransferases (HATs) decondensate chromatin and loosen the folded nucleosome, which enables the binding of transcription factors to promoter sites $[8,17,18]$. This effectively activates genes expression. Histone deacetylases (HDACs) have the opposite mechanism and effect, resulting in genes silencing. Hypoacetylation, due to either decreased HAT or increased HDAC activity, results in silencing of tumor suppressor genes. Histone hypoacetylation or HDACs overexpression has been linked to the development of prostate, breast, ovarian, colon, and gastric cancer [19]. It has been shown that HDACs are overexpressed in PDAC [20] and that this, together with HIF-la overexpression, is associated with worsening of survival prognosis [21].

In contrast to genetic mutations, which are almost impossible to reverse, epigenetic changes are potentially reversible. This implies that they are amenable to pharmacological interventions. HDAC inhibitors (HDAC-i) have shown to display antineoplastic activity in multiple tumor types, inhibiting cell growth and inducing apoptosis [17, 19]. In 2006, first HDAC-i, vorinostat (Zolinza), was approved as single-agent use in T cell lymphoma [18]. Today, several epigenetic drugs are already approved by the FDA and the EMEA for cancer treatment and around ten HDAC-is are in clinical development [22]. In PDAC cell lines usage of HDACis in combination with conventional chemotherapies has demonstrated improved antitumor activity as compared to conventional chemotherapy alone [23-25]. In vivo, however, this has not been shown. Phase II study of gemcitabine in 

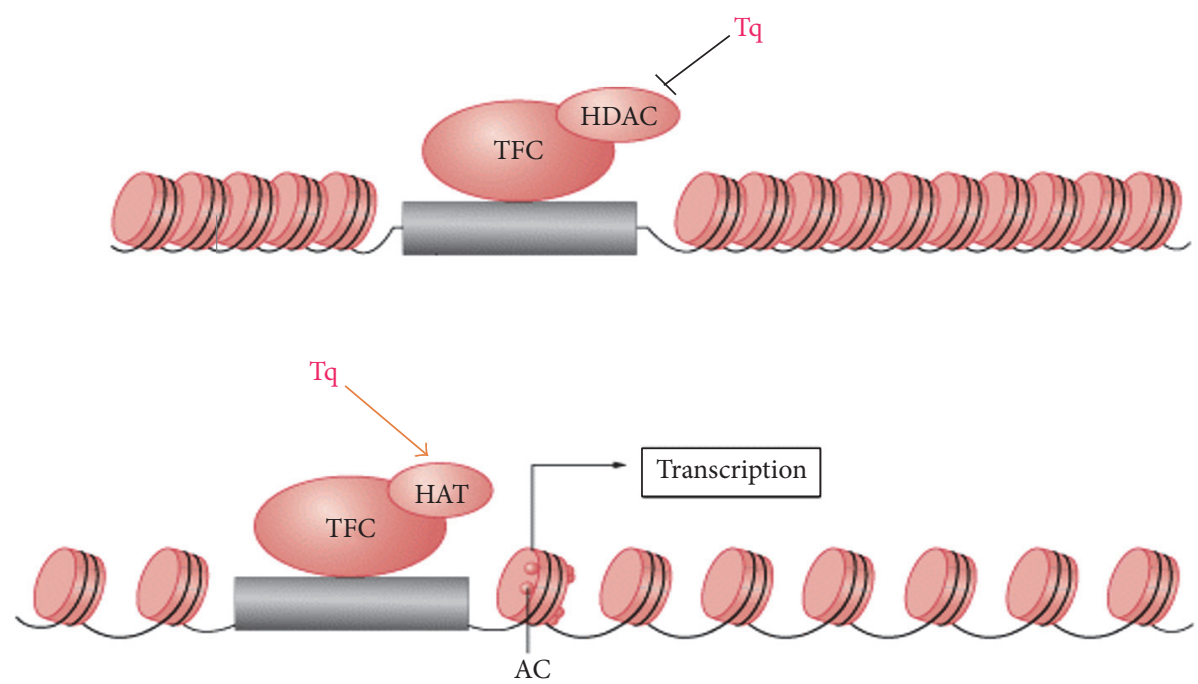

FIgURE 10: Tq actions as HDAC inhibitor. Combined inhibition of histone deacetylation and induction of histone acetylation.

combination with tacedinaline (a known HDAC-i) showed no improvement in patient response rate or survival compared to gemcitabine alone [26]. Thus search for effective HDAC-is continues.

Our study demonstrated that $\mathrm{Tq}$ could act as HDAC$i$ and could potently induce apoptosis in PDAC cells and affect the epigenetic state of histone through induction of histone acetylation and inhibition of histone deacetylation (Figure 10). Our data suggest that $\mathrm{Tq}$, as a multitarget agent, has a clinical potential that combines anti-inflammatory and proapoptotic epigenetic modification modes of action.

\section{Competing Interests}

All named authors have no financial interests in respect of this work and its publication or other interests that might be perceived to influence the results and/or discussion reported in this article.

\section{Acknowledgments}

The authors acknowledge research support and funding they have received from the Department of Surgery, Thomas Jefferson University Hospital, Philadelphia, PA, USA, and Department of Biomedical Sciences, University of New England, Biddeford, ME, USA, relevant to the work described.

\section{References}

[1] S. Padhye, S. Banerjee, A. Ahmad, R. Mohammad, and F. H. Sarkar, "From here to eternity-the secret of pharaohs: therapeutic potential of black cumin seeds and beyond," Journal of Cancer Therapy, vol. 6, pp. 495-510, 2008.

[2] S. Banerjee, S. Padhye, A. Azmi et al., "Review on molecular and therapeutic potential of thymoquinone in cancer," Nutrition and Cancer, vol. 62, no. 7, pp. 938-946, 2010.

[3] C. C. Woo, A. P. Kumar, G. Sethi, and K. H. B. Tan, "Thymoquinone: potential cure for inflammatory disorders and cancer," Biochemical Pharmacology, vol. 83, no. 4, pp. 443-451, 2012.
[4] M. P. Torres, M. P. Ponnusamy, S. Chakraborty et al., "Effects of thymoquinone in the expression of mucin 4 in pancreatic cancer cells: implications for the development of novel cancer therapies," Molecular Cancer Therapeutics, vol. 9, no. 5, pp. 14191431, 2010.

[5] M. N. Nagi, O. A. Al-Shabanah, M. M. Hafez, and M. M. Sayed-Ahmed, "Thymoquinone supplementation attenuates cyclophosphamide-induced cardiotoxicity in rats," Journal of Biochemical and Molecular Toxicology, vol. 25, no. 3, pp. 135142, 2011.

[6] O. A. Badary, M. N. Nagi, O. A. Al-Shabanah, H. A. Al-Sawaf, M. O. Al-Sohaibani, and A. M. Al-Bekairi, "Thymoquinone ameliorates the nephrotoxicity induced by cisplatin in rodents and potentiates its antitumor activity" Canadian Journal of Physiology and Pharmacology, vol. 75, no. 12, pp. 1356-1361, 1997.

[7] G. R. Leggatt and B. Gabrielli, "Histone deacetylase inhibitors in the generation of the anti-tumour immune response," Immunology and Cell Biology, vol. 90, pp. 33-38, 2011.

[8] D. M. Vigushin and R. C. Coombes, "Histone deacetylase inhibitors in cancer treatment," Anti-Cancer Drugs, vol. 13, no. 1, pp. 1-13, 2002.

[9] N. Chehl, G. Chipitsyna, Q. Gong, C. J. Yeo, and H. A. Arafat, "Anti-inflammatory effects of the Nigella sativa seed extract, thymoquinone, in pancreatic cancer cells," $H P B$, vol. 11, no. 5, pp. 373-381, 2009.

[10] R. Anandanadesan, Q. Gong, G. Chipitsyna, A. Witkiewicz, C. J. Yeo, and H. A. Arafat, "Angiotensin II induces vascular endothelial growth factor in pancreatic cancer cells through an angiotensin II type 1 receptor and ERK1/2 signaling," Journal of Gastrointestinal Surgery, vol. 12, no. 1, pp. 57-66, 2008.

[11] J. E. Chipuk, J. C. Fisher, C. P. Dillon, R. W. Kriwacki, T. Kuwana, and D. R. Green, "Mechanism of apoptosis induction by inhibition of the anti-apoptotic BCL-2 proteins," Proceedings of the National Academy of Sciences of the United States of America, vol. 105, no. 51, pp. 20327-20332, 2008.

[12] Y.-T. Hsu, K. G. Wolter, and R. J. Youle, "Cytosol-to-membrane redistribution of Bax and Bcl- $\mathrm{X}_{L}$ during apoptosis," Proceedings of the National Academy of Sciences of the United States of America, vol. 94, no. 8, pp. 3668-3672, 1997.

[13] S. Westphal and H. Kalthoff, "Apoptosis: targets in pancreatic cancer," Molecular Cancer, vol. 2, article 6, 2003. 
[14] Z.-W. Xu, H. Friess, M. W. Büchler, and M. Solioz, "Overexpression of Bax sensitizes human pancreatic cancer cells to apoptosis induced by chemotherapeutic agents," Cancer Chemotherapy and Pharmacology, vol. 49, no. 6, pp. 504-510, 2002.

[15] T. Seki, N. Ohba, R. Makino, H. Funatomi, and K. Mitamura, "Mechanism of growth-inhibitory effect of cisplatin on human pancreatic cancer cells and status of p53 gene," Anticancer Research, vol. 21, no. 3, pp. 1919-1924, 2001.

[16] R. Ju and M. T. Muller, "Histone deacetylase inhibitors activate p21(WAF1) expression via ATM," Cancer Research, vol. 63, no. 11, pp. 2891-2897, 2003.

[17] A. A. Lane and B. A. Chabner, "Histone deacetylase inhibitors in cancer therapy," Journal of Clinical Oncology, vol. 27, no. 32, pp. 5459-5468, 2009.

[18] X. Ma, H. H. Ezzeldin, and R. B. Diasio, "Histone deacetylase inhibitors: current status and overview of recent clinical trials," Drugs, vol. 69, no. 14, pp. 1911-1934, 2009.

[19] L. Lafon-Hughes, M. V. Di Tomaso, L. Méndez-Acuña, and W. Martínez-López, "Chromatin-remodelling mechanisms in cancer," Mutation Research/Reviews in Mutation Research, vol. 658, no. 3, pp. 191-214, 2008.

[20] G. Schneider, O. H. Krämer, R. M. Schmid, and D. Saur, "Acetylation as a transcriptional control mechanism-HDACs and HATs in pancreatic ductal adenocarcinoma," Journal of Gastrointestinal Cancer, vol. 42, no. 2, pp. 85-92, 2011.

[21] K. Miyake, T. Yoshizumi, S. Imura et al., "Expression of hypoxiainducible factor- $1 \alpha$, histone deacetylase 1 , and metastasisassociated protein 1 in pancreatic carcinoma: correlation with poor prognosis with possible regulation," Pancreas, vol. 36, no. 3, pp. el-e9, 2008.

[22] J. M. Wagner, B. Hackanson, M. Lübbert, and M. Jung, "Histone deacetylase (HDAC) inhibitors in recent clinical trials for cancer therapy," Clinical Epigenetics, vol. 1, no. 3-4, pp. 117-136, 2010.

[23] S. Iwahashi, M. Shimada, T. Utsunomiya et al., "Histone deacetylase inhibitor augments anti-tumor effect of gemcitabine and pegylated interferon- $\alpha$ on pancreatic cancer cells," International Journal of Clinical Oncology, vol. 16, no. 6, pp. 671-678, 2011.

[24] M. Donadelli, C. Costanzo, S. Beghelli et al., "Synergistic inhibition of pancreatic adenocarcinoma cell growth by trichostatin A and gemcitabine," Biochimica et Biophysica Acta-Molecular Cell Research, vol. 1773, no. 7, pp. 1095-1106, 2007.

[25] G. Schneider, O. H. Krämer, P. Fritsche, S. Schüler, R. M. Schmid, and D. Saur, "Targeting histone deacetylases in pancreatic ductal adenocarcinoma," Journal of Cellular and Molecular Medicine, vol. 14, no. 6 A, pp. 1255-1263, 2010.

[26] D. A. Richards, K. A. Boehm, D. M. Waterhouse et al., "Gemcitabine plus CI-994 offers no advantage over gemcitabine alone in the treatment of patients with advanced pancreatic cancer: results of a phase II randomized, double-blind, placebocontrolled, multicenter study," Annals of Oncology, vol. 17, no. 7, pp. 1096-1102, 2006. 


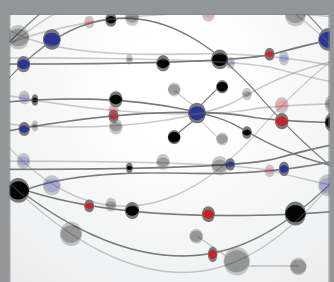

The Scientific World Journal
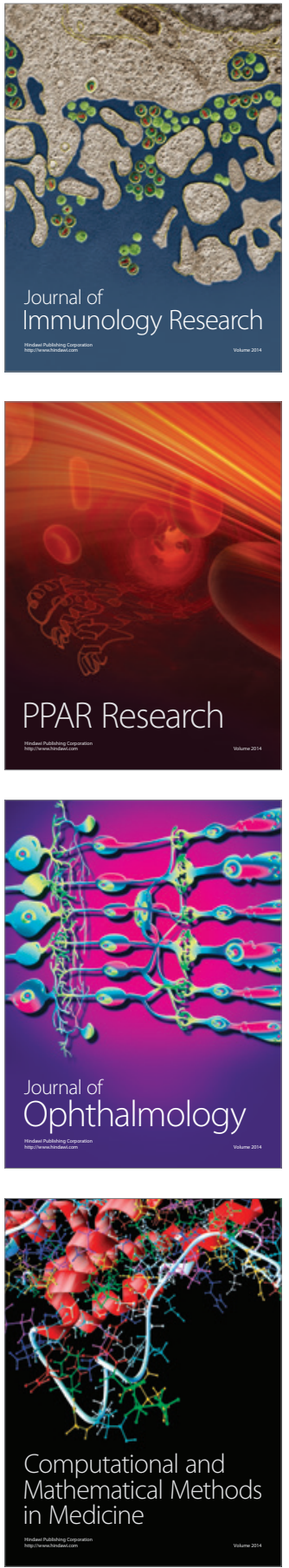

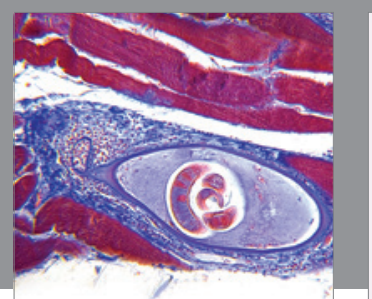

Gastroenterology Research and Practice

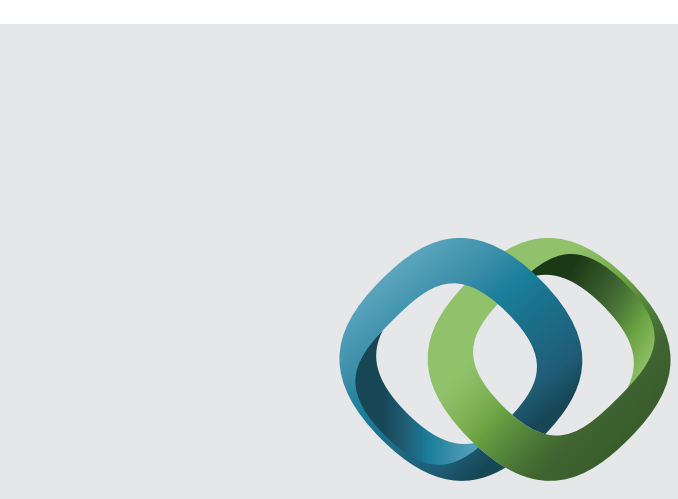

\section{Hindawi}

Submit your manuscripts at

http://www.hindawi.com
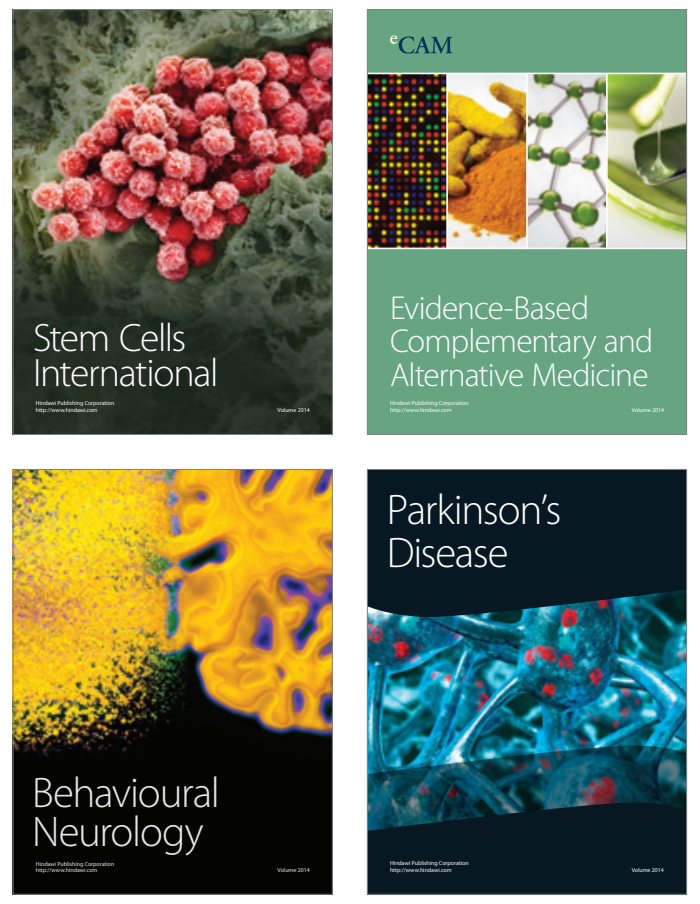
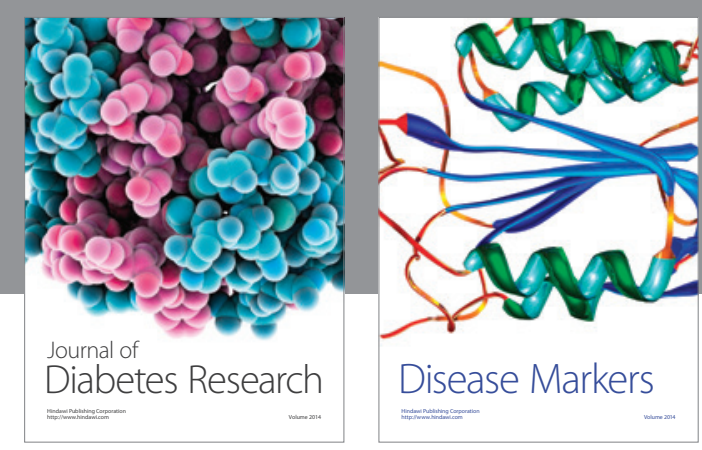

Disease Markers
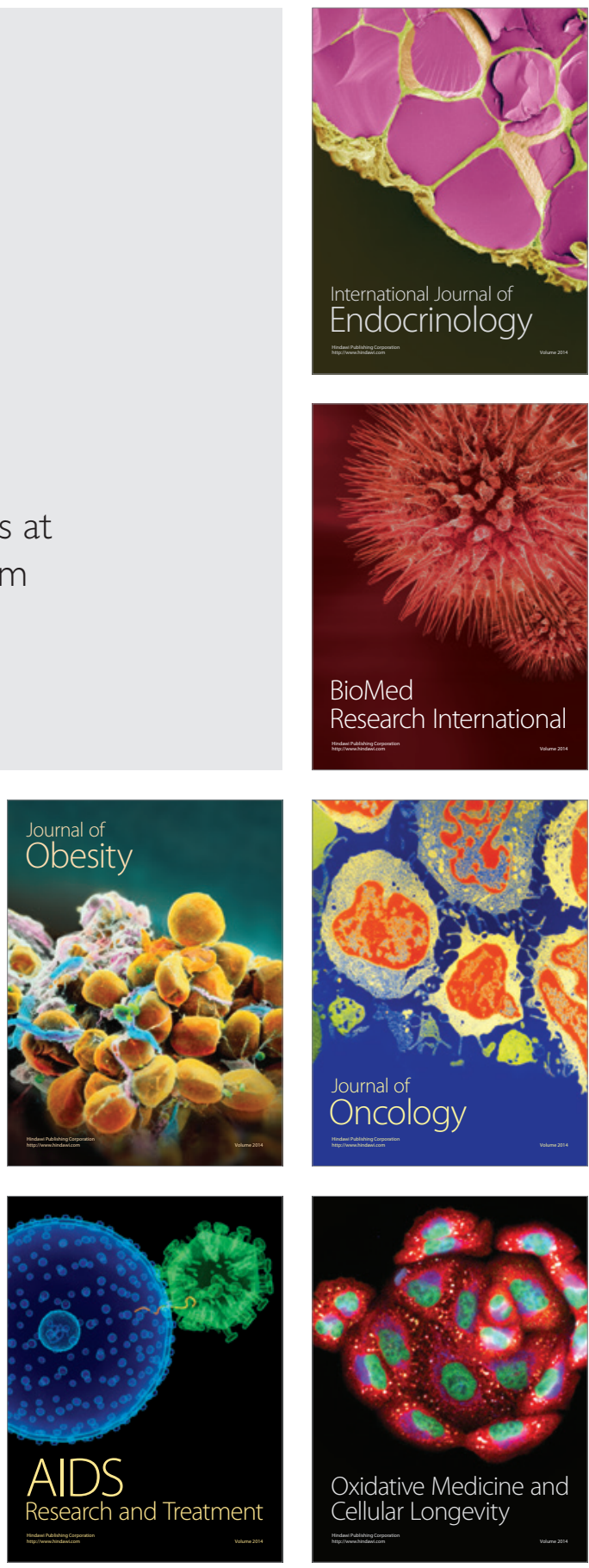